Revista de Matemática: Teoría y Aplicaciones 2000 7(1-2) : 199-216

CIMPA - UCR ISSN: 1409-2433

\title{
DYNAMICS OF A TWO-DIMENSIONAL DISCRETE-TIME SIS MODEL
}

\author{
Jaime H. Barrera* ${ }^{*}$ Ariel Cintrón-Arias ${ }^{\dagger}-$ Nicolas Davidenko ${ }^{\ddagger}$ \\ Lisa R. Denogean ${ }^{\S}$ - Saúl Ramón Franco-González $\rrbracket^{\llbracket}$
}

Received: 21 August 1999

\begin{abstract}
We analyze a two-dimensional discrete-time SIS model with a non-constant total population. Our goal is to determine the interaction between the total population, the susceptible class and the infective class, and the implications this may have for the disease dynamics. Utilizing a constant recruitment rate in the susceptible class, it is possible to assume the existence of an asymptotic limiting equation which enables us to reduce the system of two-equations into a single, dynamically equivalent equation. In this case, we are able to demonstrate the global stability of the disease-free and the endemic equilibria when the basic reproductive number $\left(R_{0}\right)$ is less than one and greater than one, respectively. When we consider a non-constant recruitment rate, the total population bifurcates as we vary the birth rate and the death rate. Using computer simulations, we observe different behavior among the infective class and the total population, and possibly, the ocurrence of a strange attractor.
\end{abstract}

Keywords: Susceptible-Infective-Susceptible (SIS), difference equations, bifurcation, basic reproductive number $\left(R_{0}\right)$, asymptotic limiting equation.

\section{Resumen}

Analizamos un modelo bidimensional SIS en tiempo discreto con una población total no constante. Nuestra meta es determinar la interacción entre la problación total, la clase susceptible y la clase infectada, y las implicaciones que esto puede tener para la dinámica de la enfermedad. Usando una tasa de reclutamiento constante en la

\footnotetext{
${ }^{*}$ Cornell University, jhb9149@hotmail.com

†657 Rhodes Hall, Cornell University, Ithaca, NY 14853-3801, U.S.A. E-Mail: ariel@cam.cornell.edu

${ }^{\ddagger}$ Harvard University, U.S.A. E-Mail: ndavidenko@yahoo.com

${ }^{\S}$ C.A.M., Cornell University, U.S.A. E-Mail: lisa_denogean@hotmail.com

ฯUniversity of California at Irvine, E-Mail: francos@uci.edu
} 
clase susceptible, es posible asumir la existencia de una ecuación limitante asintótica que permite reducir el sistema de dos ecuaciones a una sola ecuación dinámicamente equivalente. En este caso, somos capaces de demostrar la estabilidad global de los equilibrios libres de enfermedad y la endemia, cuando el número básico reproductivo $\left(R_{0}\right)$ es menor que uno y mayor que uno, respectivamente. Cuando se considera una tasa de reclutamiento no constante, la problacón total se bifurca cuando se varía la tasa de natalidad y la tasa de mortalidad. Usando simulaciones computacionales, observamos diferentes comportamientos entre la clase infectada y la población total, y posiblemente, la ocurrencia de un extraño atractor.

Palabras clave: Susceptible-Infeccioso-Susceptible (SIS), ecuaciones en diferencias, bifurcación, número básico reproductivo $\left(R_{0}\right)$, ecuación limitante asintótica.

Mathematics Subject Classification: 92D30, 92B15

\section{Introduction}

Infectious diseases are classified into two groups: microparasitic diseases which are transmitted by viruses and bacteria, and macroparasitic diseases which are are transmitted by worms. Microparasites can reproduce within their host and the diseases can be transmitted via one host to the other. On the other hand, macroparasites show very complicated life-cycles, and usually involve a secondary host, or carrier, as explained by Anderson [3].

One problem associated with the modelling of infectious diseases is that it is very difficult to measure or even estimate the total infectious population. A second problem is that primitive models assume that viruses propagate freely and randomly encounter new hosts. However, the spread of microparasitic diseases is actually caused by contact or close interaction between the infected and healthy individuals. In all microparasitic epidimiological models, the distinction is made between the disease-carrying individuals (the infective class), and the disease-free individuals (the susceptible class)(Edelstein-Keshet)[8]. In a Susceptible-Infective-Susceptible $(S I S)$ model, formerly infective individuals who are cured do not develop permanent immunity, but instead return to the susceptible class (Allen) [2].

This research investigates a discrete-time $S I S$ model which may be applicable to particular diseases (Allen L.) [1], or may be considered as a discrete analog of the more well-known continuous-time Markov models. In our discrete time $(S I S)$ model, the total population is not constant. We call the equation for the total population the demographic equation. For this model, we separate our work into two cases: a constant and non-constant recruitment (or birth) rate.

For the case of a constant recruitment rate, we assume there exists a limiting equation that enables us to reduce the system of two equations to a single equation. We prove that, under the condition that the basic reproductive number $\left(R_{0}\right)$ is less than one, the disease-free equilibrium is globally stable. Also, when $R_{0}$ is greater than one we prove that the endemic equilibrium is globally stable.

In the case where we consider non-constant recruitment, there are bifurcations for the demographic equation. These bifurcations are governed by varying the birth and death 
rates. In the range of parameters where there exists a positive fixed point for the demographic equation, we can reduce to a single equation (as in the constant recruitment). Then we can obtain global stability of the disease-free and endemic equilibria for the infective equation. Outside the range of parameters where we can reduce to a single equation, we perform computational analysis through bifurcation diagrams for the demographic, susceptible, and infective equations.

We use the following model:

$$
\begin{aligned}
S_{n+1} & =f\left(T_{n}\right)+S_{n} \pi(n, n+1) h\left(I_{n}\right)+I_{n} \pi(n, n+1)[1-\xi(n, n+1)] \\
I_{n+1} & =S_{n} \pi(n, n+1)\left[1-h\left(I_{n}\right)\right]+I_{n} \pi(n, n+1) \xi(n, n+1) \zeta(n, n+1),
\end{aligned}
$$

with

$$
T_{n}=S_{n}+I_{n}=f\left(T_{n}\right)+T_{n} \pi(n, n+1)+I_{n} \pi(n, n+1)[\zeta(n, n+1)-1]
$$

where from generation $\mathrm{n}$ to $\mathrm{n}+1$,

$$
\begin{aligned}
1-\pi(n, n+1) & =1-e^{-\mu} \text { is the probability of death due to natural causes, } \\
1-\xi(n, n+1) & =1-e^{-\sigma} \text { is the probability of recovering, } \\
1-\zeta(n, n+1) & =1-e^{-\rho} \text { is the probability of death due to infection } \\
h\left(I_{n}\right) & =e^{-\alpha I_{n}} \text { is the probability of not becoming infected, } \\
f\left(T_{n}\right) & =\text { birth or immigration rate ( } 2 \text { cases }),
\end{aligned}
$$

and $\alpha, \mu, \sigma>0$. However, we consider cases for $\rho \geq 0$ to account for infections which may or may not be fatal. In the majority of our analysis we take $\rho=0$.

\subsection{Assumptions}

The following assumptions are primarily biologically motivated:

1. The time step is one generation.

2. There is no negative population.

3. From generation $n$ to $n+1$, infections occur before deaths.

4. Rates of events occur independent of generation.

5. There are no infected births-all births enter into the susceptible class.

6. In the case of the non-constant recruitment if there are no people, then there are no births: $f(0)=0$.

7. If there are too many people, then there are not enough resources to sustain further births:

$$
\lim _{T_{n} \rightarrow \infty} f\left(T_{n}\right)=0 .
$$

8. The probability of not becoming infected when there are no people is one: $h(0)=1$. 
9. The probability of not becoming infected as the number of infected increases is a strictly decreasing function: $h^{\prime}\left(I_{n}\right)<0$.

10. With too many infectious people, the probability of not becoming infected is zero:

$$
\lim _{I_{n} \rightarrow \infty} h\left(I_{n}\right)=0 .
$$

\section{Constant recruitment rate}

We use constant recruitment to model a constant flow of new susceptibles through either birth or immigration. Here,

$$
f\left(T_{n}\right)=\Lambda>0
$$

So, our new model becomes:

$$
\begin{aligned}
S_{n+1} & =\Lambda+S_{n} e^{-\mu} e^{-\alpha I_{n}}+I_{n} e^{-\mu}\left[1-e^{-\sigma}\right], \\
I_{n+1} & =S_{n} e^{-\mu}\left[1-e^{-\alpha I_{n}}\right]+I_{n} e^{-\mu} e^{-\sigma},
\end{aligned}
$$

and

$$
T_{n+1}=\Lambda+T_{n} e^{-\mu}
$$

\subsection{Limiting equation}

$T_{n}$ is linear and the solution to $(3)$ is,

$$
T_{n}=e^{-\mu n}\left(T_{0}-\frac{\Lambda}{1-e^{-\mu}}\right)+\frac{\Lambda}{1-e^{-\mu}}
$$

Notice that

$$
\lim _{n \rightarrow \infty} T_{n}=\frac{\Lambda}{1-e^{-\mu}} \equiv T_{\infty} .
$$

We set $T_{0}=T_{\infty}$. This simply means that the population starts at the asymptotic limit of the demographic population. Now, we plug $S_{n}=T_{\infty}-I_{n}$ into (2) to get:

$$
v\left(I_{n}\right)=\left(T_{\infty}-I_{n}\right) e^{-\mu}\left[1-e^{-\alpha I_{n}}\right]+I_{n} e^{-(\mu+\sigma)} .
$$

The function $v$ is a single-humped function. Figures 1-3 show some graphs of this function for various parameters. It is apparent from graphs of $v$ (and could be easily shown using calculus) that $v\left(I_{n}\right)=0$ has one positive root, $I_{r}$. As $v\left(I_{n}\right)=0$ is a transcendental equation, a formula for $I_{r}$ could not be found explicitly. In consideration of assumption 2, we now define our infectious population equation:

$$
u\left(I_{n}\right)=I_{n+1}= \begin{cases}v\left(I_{n}\right) & , \text { if } I_{n} \leq I_{r} \\ 0 & , \text { if } I_{n}>I_{r}\end{cases}
$$




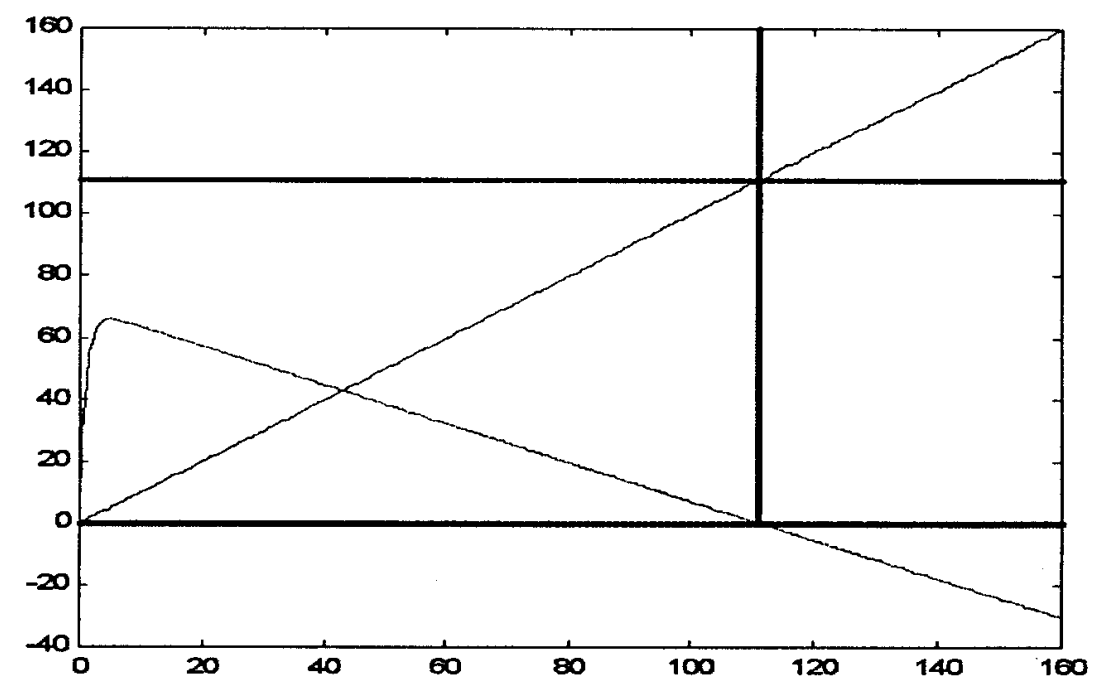

Figure 1: $v$ for various parameters with the line $y=x: \alpha=1, \sigma=1, \lambda=0.7, \mu=0.01$, $R_{0}=109.5512>1$.

We wish to consider only the dynamics for $I_{n} \leq I_{r}$. The proofs to follow assume that our function does not become degenerate such that a great proportion of initial conditions get mapped out of the region $\left[0, I_{r}\right]$. This happens if the maximum of $u$, denoted by $M$, is greater then $I_{r}$, i.e.

$$
I_{r} \leq M
$$

Exercise 11.4.6 in Strogatz [12] will lead the reader to the realization that if inequality (7) is true, then the set of $I_{0}$ for which trajectories do not get mapped to zero after some finite amount of iterations is topologically equivalent to a Cantor set. The proof that there exists a globally attracting endemic point rests on the assumption that inequality (7) is not true. More about this will be said when it becomes relavent.

So we have reduced the system (1-2) of two equations to a single equation. We use the dynamics of this discrete-time single equation to draw conclusions about the dynamics of the two-equation system. This is not an unusual comparison in analysis of systems which have a limiting demographic state. This reduction to a single equation can also be justified by our simulations in which the system and the single equation exhibit the same qualitative dynamics. Theoretical results for continuous-time systems have been obtained by Theime \& Castillo-Chavez [4].

\section{$2.2 \quad R_{0}$}

We can see that $I^{*}=0$ is a fixed point of (6). We determine $R_{0}$ for the disease-free equilibrium by using $\left|u^{\prime}(0)\right|<1$. This yields our basic reproductive number,

$$
R_{0}=\frac{\alpha T_{\infty} e^{-\mu}}{1-e^{-(\mu+\sigma)}}
$$




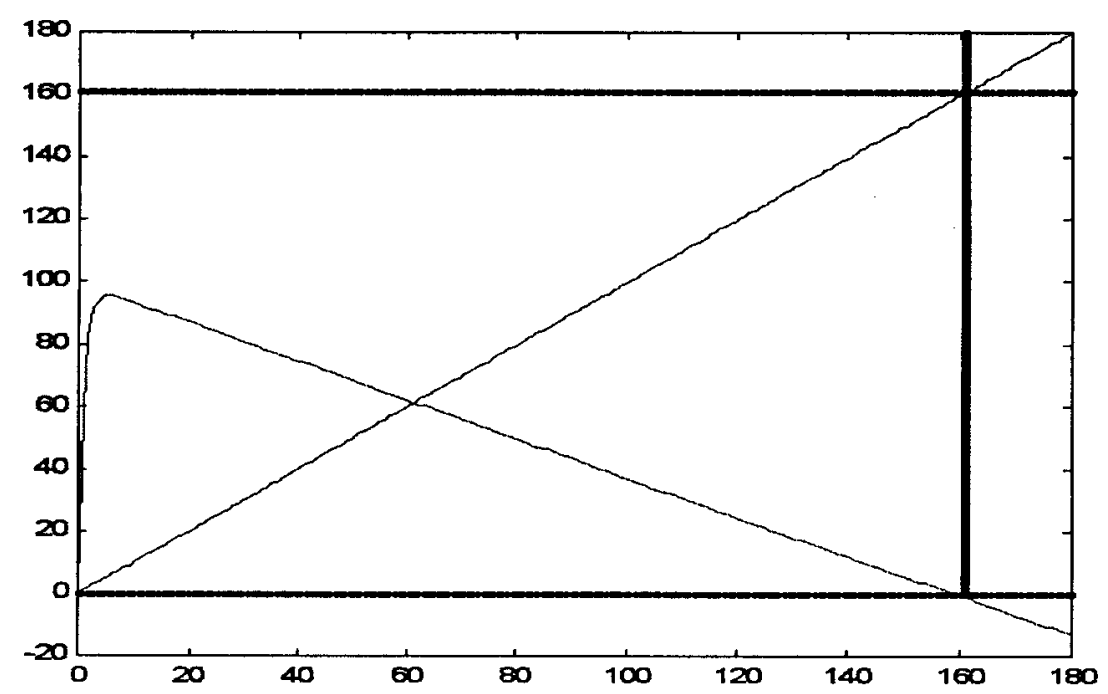

Figure 2: $v$ for various parameters with the line $y=x: \alpha=1, \sigma=1, \lambda=1, \mu=0.01$, $R_{0}=156.5017>1$.

Biologically, this $R_{0}$ represents the average number of effective contacts multiplied by the number of available susceptibles. This is found by observing that $\frac{\alpha}{1-e^{-(\mu+\sigma)}}$ is the infection rate per infective per generation multiplied by the average number of generations an individual is infectious before dying or recovering. So, this yields the average number of effective contacts. $T_{\infty} e^{-\mu}$ is the population size when there is no infection, discounted by deaths. So, this $R_{0}$ is interpreted as the number of people an infected person infects. We will see that if $R_{0}>1$, the disease can invade, and if $R_{0}<1$, the disease will die out.

\subsection{Dynamics of single equation, case $R_{0}<1$}

We will now show that for certain stability conditions, $I^{*}=0$ is a global attractor.

Lemma 2.1 Assume that $f(x)$ is a continuous function of $x$. If the solution of the difference equation $x_{n+1}=f\left(x_{n}\right)$ is convergent, then the limit of $x_{n}$ is a fixed point of $f(x)$. Hence $\bar{x}$ is a fixed point of $f(x)$.

PROOF: Given

$$
x_{n+1}=f\left(x_{n}\right)
$$

by taking the limit in both sides from $n \rightarrow \infty$

$$
\lim _{n \rightarrow \infty} x_{n+1}=\lim _{n \rightarrow \infty} f\left(x_{n}\right)
$$

Let $\bar{x}=\lim _{n \rightarrow \infty} x_{n+1}$, then $\bar{x}=\lim _{n \rightarrow \infty} f\left(x_{n}\right)=f\left(\lim _{n \rightarrow \infty} x_{n}\right)=f(\bar{x})$.

Hence, $\bar{x}$ is a fixed point of $\mathrm{f}(\mathrm{x})$. 


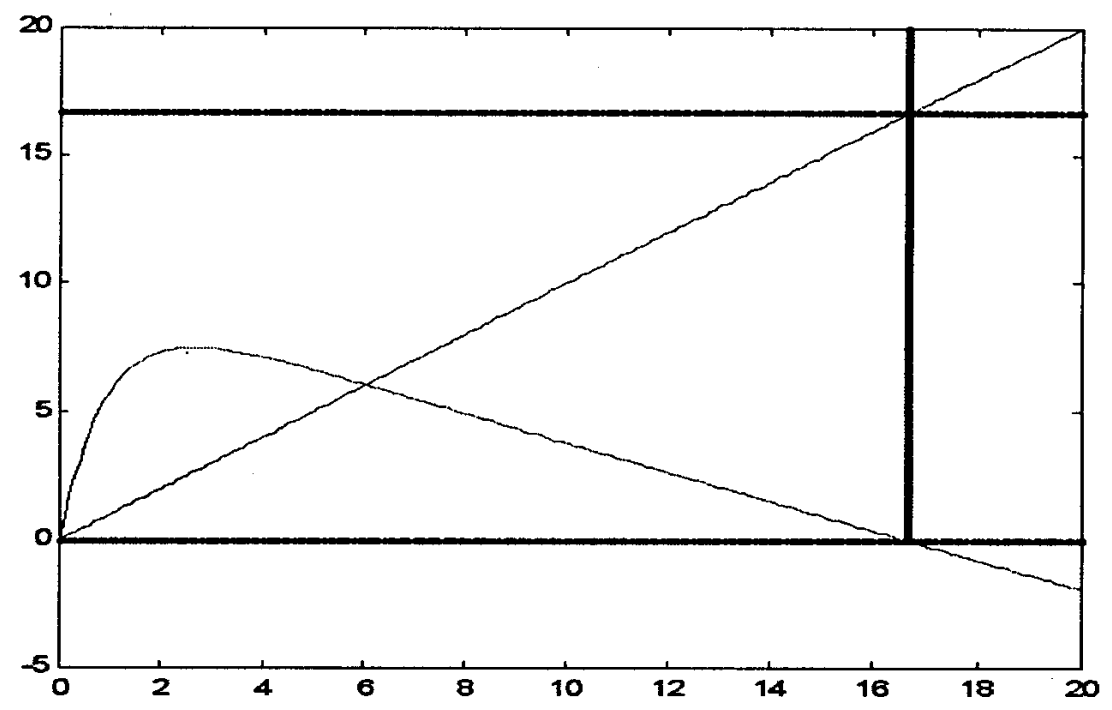

Figure 3: $v$ for various parameters with the line $y=x: \alpha=1, \sigma=1, \lambda=1, \mu=0.1$, $R_{0}=14.2526>1$.

Theorem 2.1 If $R_{0}<1$, then $I^{*}=0$ is a global attractor of $u\left(I_{n}\right)$.

Proof: By definition, $I^{*}$ is globally stable if

$$
\lim _{n \rightarrow \infty} I_{n}=I^{*}
$$

for all $I_{0} \in[0, \infty)$. Clearly, $I^{*}=0$ is a fixed point of $(6)$. We have to show that $I^{*}$ is unique and that all initial conditions tend towards $I^{*}$. We will first show that $I_{n+1}<I_{n}$ for all $n \in \mathbb{N}$. First, define:

$$
\begin{gathered}
f:[0, \infty) \rightarrow \mathbb{R}, \\
f(x)=1-e^{-\alpha x}-\alpha x,
\end{gathered}
$$

where $\alpha>0$ and $f$ is continuous.

$$
\begin{aligned}
f(0) & =0 \\
f^{\prime}(x) & =\alpha e^{-\alpha x}-\alpha=\alpha\left(e^{-\alpha x}-1\right) \\
\text { Since }-\alpha x & <0 \text { by definition, } \\
\Rightarrow e^{-\alpha x} & <1 \\
e^{-\alpha x}-1 & <0 \\
\Rightarrow f^{\prime}(x) & <0
\end{aligned}
$$

Therefore, $f(x)$ is decreasing. So, for all $a \in(0, \infty)$,

$$
\begin{aligned}
f(a) & <f(0) \\
\text { By }(9), f(a) & <0
\end{aligned}
$$




$$
\begin{aligned}
\Rightarrow f(x) & <0, \text { for all } x>0 \\
\Rightarrow 1-e^{-\alpha I_{n}}-\alpha I_{n} & <0 \\
1-e^{-\alpha I_{n}} & <\alpha I_{n} \\
\frac{1}{\alpha I_{n}} & <\frac{1}{1-e^{-\alpha I_{n}}} \\
\Rightarrow \frac{1-e^{-(\mu+\sigma)}}{\alpha I_{n}} & <\frac{1-e^{-(\mu+\sigma)}}{1-e^{-\alpha I_{n}}}
\end{aligned}
$$

Now,

$$
\begin{aligned}
R_{0} & =\frac{\alpha T_{\infty} e^{-\mu}}{1-e^{-(\mu+\sigma)}}<1 \\
\Rightarrow e^{-\mu}\left(e^{-\sigma}+\frac{\alpha \Lambda}{1-e^{-\mu}}\right) & <1 \\
\Rightarrow e^{-\mu}\left(\frac{1}{I_{n}} \frac{\Lambda}{1-e^{-\mu}}-1\right) & <\frac{1}{I_{n}}\left(\frac{1-e^{-(\mu+\sigma)}}{\alpha}\right)-e^{-\mu} \\
& \left.<\frac{1-e^{-(\mu+\sigma)}}{\alpha I_{n}}\right) \\
\text { From }(10), \Rightarrow & <\frac{1-e^{-(\mu+\sigma)}}{1-e^{-\alpha I_{n}}} \\
\text { Therefore, } e^{-\mu}\left(\frac{1}{I_{n}} \frac{\Lambda}{1-e^{-\mu}}-1\right) & <\frac{1-e^{-(\mu+\sigma)}}{1-e^{-\alpha I_{n}}} \\
\frac{1}{I_{n}} e^{-\mu}\left(\frac{\Lambda}{1-e^{-\mu}}-I_{n}\right)\left(1-e^{-\alpha I_{n}}\right) & <1-e^{-(\mu+\sigma)} \\
\frac{1}{I_{n}} e^{-\mu}\left(T_{\infty}-I_{n}\right)\left(1-e^{-\alpha I_{n}}\right)+e^{-(\mu+\sigma)} & <1 \\
e^{-\mu}\left(T_{\infty}-I_{n}\right)\left(1-e^{-\alpha I_{n}}\right)+I_{n} e^{-(\mu+\sigma)} & <I_{n} \\
\Rightarrow I_{n+1} & <I_{n}
\end{aligned}
$$

Therefore, we have a strictly decreasing series.

$$
\begin{aligned}
\text { Furthermore, since } I_{n+1} & <I_{n}, \\
\Rightarrow u\left(I_{n}\right) & <I_{n} \\
\Rightarrow u\left(I_{n}\right) & \neq I_{n}\left(\text { for all } I_{n} \neq 0 .\right)
\end{aligned}
$$

Therefore, $I^{*}=0$ is the only fixed point, and we have uniqueness.

So, $u\left(I_{n}\right)$ is continuous, decreasing, and bounded below by zero. Thus, $u\left(I_{n}\right)$ must converge, and by the above lemma,

$$
\lim _{n \rightarrow \infty} I_{n}=I^{*}
$$

Therefore, $I^{*}=0$ is global attractor. 
When $R_{0}>1$ we need not worry that we will end up with only a dust-like Cantor set which goes to the endemic attractor. We can see from figures 1-3 that inequality (7) is not satisfied for these choices of parameter values. Furthermore, simulations confirm that no choice of parameters $\left(R_{0}<1\right.$ or $\left.R_{0} \geq 1\right)$ give us inequality $(7)$.

\subsection{Dynamics of single equation, case $R_{0}>1$}

In the case $R_{0}>1$, the disease-free equilibrium is unstable. We will show that there is a stable, unique endemic fixed point that attracts all interior points for values of $R_{0}>1$. This $I^{*}$ satisfies $u\left(I^{*}\right)=I^{*}$ such that $I^{*}>0$, and $\lim _{n \rightarrow \infty} I_{n}=I^{*}$ for all $I_{0}>0$ and $R_{0}>1$.

First, we will show the existence and uniqueness of $I^{*}>0$ for $R_{0}>1$.

Theorem 2.2 There exists a fixed point $I^{*}>0$ of $u\left(I_{n}\right)$ for $R_{0}>1$.

PROOF:

$$
\begin{aligned}
R_{0} & =\frac{\alpha T_{\infty} e^{-\mu}}{1-e^{-\mu+\sigma}}>1 \\
\Rightarrow \alpha T_{\infty} e^{-\mu} & >1-e^{-(\mu+\sigma)}
\end{aligned}
$$

Let

$$
\begin{aligned}
g(x)= & u(x)-x \\
= & T_{\infty} e^{-\mu}-e^{-\mu}\left(T_{\infty}-x\right) e^{-\alpha x} \\
& -\left(e^{-\mu}-e^{-(\mu+\sigma)}+1\right) x \\
= & 0 \text { for a fixed point of } u(x) \\
\text { Obviously, } g(0)= & 0, \text { but find } I^{*}>0 \\
g^{\prime}(x)= & -\left(e^{-\mu}-e^{-(\mu+\sigma)}+1\right)+e^{-\mu}\left(\alpha T_{\infty}+1\right) e^{-\alpha x} \\
& -\alpha x e^{-\mu} e^{-\alpha x} \\
g^{\prime}(0)= & -\left(1-e^{-(\mu+\sigma)}\right)+\alpha T_{\infty} e^{-\mu} \\
\Rightarrow g^{\prime}(0)> & 0 \text { by }(11)
\end{aligned}
$$

So, $g(x)$ strictly increases from $x=0$.

By 12, we have

$$
\begin{aligned}
\lim _{x \rightarrow \infty} g(x) & =e^{-\mu} T_{\infty}-\lim _{x \rightarrow \infty}-\left(e^{-\mu}-e^{-(\mu+\sigma)}+1\right) x \\
\text { Note that } e^{-\mu}+1 & >e^{-(\mu+\sigma)} \\
\text { So, } \lim _{x \rightarrow \infty} g(x) & =-\infty
\end{aligned}
$$

By the intermediate value theorem, $g(x)$ has at least one positive zero since $g(x)$ is continuous. Therefore, there exists $I^{*}>0$ such that $u\left(I^{*}\right)=I^{*}$.

Theorem 2.3 $I^{*}>0$ is a unique positive fixed point of $u\left(I_{n}\right)$ for $R_{0}>1$. 
PROOF: Let $g(x)=u(x)-x$ as before. We have proved that there is at least one positive fixed point of $u(x) . I^{*}>0$ is unique if there is only one $x^{*}>0$ such that $g^{\prime}\left(x^{*}\right)=0$, since $g(0)=0, g^{\prime}(0)>0$, and $\lim _{x \rightarrow \infty} g(x)=-\infty$.

$$
\begin{aligned}
\text { Now, by }(13), \lim _{x \rightarrow \infty} g^{\prime}(x) & =-\left(e^{-\mu}-e^{-(\mu+\sigma)}+1\right), \\
\text { by (14) } \lim _{x \rightarrow \infty} g^{\prime}(x) & <0 .
\end{aligned}
$$

So, since $g^{\prime}(x)$ is continuous, by the intermediate value theorem there exists at least one $x^{*}$ such that $g^{\prime}\left(x^{*}\right)=0$.

Look for extrema of $g^{\prime}(x)$ :

$$
\begin{aligned}
g^{\prime \prime}\left(x^{* *}\right) & =-\alpha e^{-\mu}\left(1+\alpha T_{\infty}\right) e^{-\alpha x^{* *}}-\alpha x^{* *} e^{-\mu} e^{-\alpha x^{* *}}+\alpha^{2} x^{* *} e^{-\mu} e^{-\alpha x^{* *}}=0 \\
\Rightarrow \alpha x^{* *} & =2+\alpha T_{\infty} \\
x^{* *} & =T_{\infty}+\frac{2}{\alpha}
\end{aligned}
$$

And, $g^{\prime \prime}(x)>0$ if $x>x^{* *}$$$
g^{\prime \prime}(x)<0 \text { if } x<x^{* *}
$$

Thus, there is one local extrema of $g^{\prime}(x)$, namely $g^{\prime}\left(x^{* *}\right)$.

$$
\text { Now, } \begin{aligned}
g^{\prime}\left(x^{* *}\right) & =e^{-\mu}\left(1+\alpha T_{\infty}\right) e^{-\alpha x^{* *}}-\left(\alpha T_{\infty}+2\right) e^{-\mu} e^{-\alpha x^{* *}}-\left(e^{-\mu}-e^{-(\mu+\sigma)}+1\right) \\
& =e^{-\mu} e^{-\alpha x^{* *}}(-1)-\left(e^{-\mu}-e^{-(\mu+\sigma)}+1\right) \\
& <0 \text { by }(14)
\end{aligned}
$$

So, the minimum occurs at $g^{\prime}\left(x^{* *}\right)$, and $g^{\prime}(x)$ increases asymptotically to a negative number in the interval $\left(x^{* *}, \infty\right)$, since $\lim _{x \rightarrow \infty} g^{\prime}(x)<0$. And, $g^{\prime}(x)$ strictly decreases in the interval $\left(0, x^{* *}\right)$. Therefore, there is only one zero of $g^{\prime}(x)$ in the interval $\left(0, x^{* *}\right)$.

This implies that the function $g(x)$ has only one relative extrema. Therefore, there is only one $x^{*}>0$ such that $g\left(x^{*}\right)=0$. This is the one and only $I^{*}>0$ such that $u\left(I^{*}\right)=I^{*}$. -We know for $R_{0}>1, I^{*}=0$ is unstable. If there are no $m$-cycles $(m \geq 1)$, then we can conclude that $I^{*}>0$ is globally stable within a certain range of $R_{0}$.

Theorem 2.4 $I^{*}>0$ is globally stable for $1<R_{0}<R^{*}$.

PROOF: (By contradiction)

There exists an $R^{*}$ such that for $R_{0}<R^{*}$,

$$
\begin{aligned}
& T_{\infty} \geq I \text { for all I } \\
& \Rightarrow T_{\infty} \alpha e^{-\mu} e^{-\alpha I} \geq I \alpha e^{-\mu} e^{-\alpha I} \\
& \alpha T_{\infty} e^{-\mu} e^{-\alpha I}-\alpha I e^{-\mu} e^{-\alpha I} \geq 0>e^{-\mu}-e^{-\mu} e^{-\sigma}-e^{-\mu-\alpha I}-1 \\
& \Rightarrow \alpha T_{\infty} e^{-\mu} e^{-\alpha I}-\alpha I e^{-\mu} e^{-\alpha I}>-e^{-\mu} e^{-\sigma}-e^{-\mu} e^{-\alpha I}-1+e^{-\mu} \\
& 1-e^{-\mu}+\alpha T_{\infty} e^{-\mu} e^{-\alpha I}+e^{-\mu} e^{-\alpha I}-\alpha I e^{-\mu} e^{-\alpha I}+e^{-\mu} e^{-\sigma}>0 \\
& \Rightarrow 1+u^{\prime}(I)>0
\end{aligned}
$$


Where,

$$
\begin{aligned}
u(I) & =\left(T_{\infty}-I\right)\left(1-e^{-\alpha I}\right) e^{-\mu}+I e^{-\mu} e^{-\sigma} \\
u^{\prime}(I) & =-e^{-\mu}+\alpha T_{\infty} e^{-\mu} e^{-\alpha I}+e^{-\mu} e^{-\alpha I}-\alpha I e^{-\mu} e^{-\alpha I}+e^{-\mu} e^{-\sigma}
\end{aligned}
$$

Suppose two-cycle such that $u\left(I_{1}\right)=I_{2}$ and $u\left(I_{2}\right)=I_{1}$.

We have $1+u^{\prime}(I)>0$

$$
\begin{array}{r}
\Rightarrow \int_{I_{1}}^{I_{2}}\left(1+u^{\prime}(I)\right) d I>0 \\
\Rightarrow 0<\int_{I_{1}}^{I_{2}}\left(1+u^{\prime}(I)\right) d I=I_{2}+u\left(I_{2}\right)-I_{1}-u\left(I_{1}\right)=0
\end{array}
$$

So there cannot exist a two-cycle. Then by Cull [5] and Allen [1] no two-cycles implies no m-cycles. This is by assumption that the set is invariant. Thus, we have global stability.

\section{Non-constant recruitment model}

This case models the effect of replacing constant recruitment with a Ricker equation. Here,

$$
\begin{gathered}
\qquad\left(T_{n}\right)=\Lambda=\beta T_{n} e^{-\gamma T_{n}} \\
S_{n+1}=\beta T_{n} e^{-\gamma T_{n}}+S_{n} e^{-\alpha I_{n}} e^{-\mu}+e^{-\mu}\left[1-e^{-\sigma}\right] I_{n} \\
I_{n+1}=S_{n} e^{-\mu}\left[1-e^{-\alpha I_{n}}\right]+I_{n} e^{-\mu} e^{-\sigma} \\
T_{n+1}=S_{n+1}+I_{n+1}=\beta T_{n} e^{-\gamma T_{n}}+T_{n} e^{-\mu} \\
\text { where } \beta=\text { maximal birth rate/person/generation }
\end{gathered}
$$

\subsection{Demographic equation}

Equation (18) is called the demographic equation. We analyze the dynamics of (18) in order to determine sufficient and necessary conditons for stability of the fixed points. Let $f_{1}(T)=\beta T e^{-\gamma T}+T e^{-\mu}$ then equilibria of the demographic equation are given by the solutions of the following equation,

$$
f_{1}(T)=T
$$

so the fixed points are as follows,

$$
\begin{gathered}
T_{\infty}^{1}=0 \\
T_{\infty}^{2}=\frac{1}{\gamma} \ln \left(\frac{\beta}{1-e^{-\mu}}\right)
\end{gathered}
$$


Let $R_{d}=\frac{\beta}{1-e^{-\mu}}$. Now notice that if $R_{d}<1$ then there is no positive fixed point. In this case the only fixed point is $T_{\infty}=0$. Therefore, this equilibrium point is locally stable by the following statement:

$$
\begin{array}{r}
R_{d}<1 \\
\Rightarrow \beta<1-e^{-\mu} \\
\Rightarrow 0<\beta+e^{-\mu}<1 \\
\Rightarrow\left|f_{1}^{\prime}(0)\right|<1
\end{array}
$$

On the other hand, in the case where $R_{d}>1$, there exists two fixed points.

Lemma 3.1 For $1<R_{d}<e^{\frac{2}{1-e^{-\mu}}}, T_{\infty}^{2}$ is locally stable.

PROOF:

$$
\begin{array}{r}
1<R_{d}<e^{\frac{2}{1-e^{-\mu}}} \\
\Rightarrow-2<-\left(1-e^{-\mu}\right) \ln \left(R_{d}\right)<0 \\
\Rightarrow\left|1-\left(1-e^{-\mu}\right) \ln \left(R_{d}\right)\right| \\
\Rightarrow\left|f_{1}^{\prime}\left(\frac{1}{\gamma} \ln \left(R_{d}\right)\right)\right|<1 \quad \square
\end{array}
$$

The biological interpretation of $R_{d}$ explains the importnce of the parameters $\beta$ and $\mu$ in the stability of the population. $\beta$ is the maximal birth rate/person/generation- this occurs when $T_{\infty}$ is small and there is no competition for resources. $\frac{1}{1-e^{-\mu}}$ is the average number of generations an individual is alive before dying, that is, the average number of generations in which an individual can reproduce. So, $R_{d}$ is the average number of births per person that enter the system during the individual's lifetime. We show that $R_{d}>1$ implies stability of a non-zero population. Larger $R_{d}$ values cause chaotic behavior in the demographic equation.

\subsection{Limiting equation $I_{n+1}$ : case $R_{d}<1$}

As we have already seen previously, $R_{d}<1$ implies the existence of only one fixed point. The limiting equation is not used in this case because that would imply the extinction of the population.

\subsection{Limiting equation $I_{n+1}$ : case $1<R_{d}<e^{\frac{2}{1-e^{-\mu}}}$}

Assuming (21), we can reduce (16) and (17) into a single equation by utilizing the limiting equation. Then we have the following equation:

$$
I_{n+1}=\left(T_{\infty}-I_{n}\right) e^{-\mu}\left(1-e^{-\alpha I_{n}}\right)+I_{n} e^{-\mu} e^{-\sigma}
$$


where $T_{\infty}=\frac{1}{\gamma} \ln \left(R_{d}\right)$.

Again by studying the stability conditions for the disease free equilibrium, we get the basic reproductive number:

$$
R_{0}=\frac{\alpha e^{-\mu} \frac{1}{\gamma} \ln \left(R_{d}\right)}{1-e^{-(\mu+\sigma)}}
$$

Therefore, by the theorem of section 2.3 , the disease free equilibrium of (22) is a global attractor if $R_{0}<1$. In addition, if $R_{0}>1$ then the endemic equilibrium of (22) is a global attractor.

\section{$3.4 R_{d}>e^{\frac{2}{1-e^{-\mu}}}$}

$T_{n}$ undergoes a period-doubling route to chaos. In this case, a $T_{\infty}$ substitution would not be justified, as $T_{n}$ bifurcates into multiple $T_{\infty}$ values. We investigate the dynamics of the system using computer simulations.

The Appendix includes bifurcation plots of the system with different parameters. The point at which the 2-cycle occurs is when $R_{d}$ becomes greater than $e^{\frac{2}{1-e^{-\mu}}}$.

In Figure 5, we have $\alpha=0.5, \beta=15, \gamma=1, \sigma=0.1, S_{0}=5, I_{0}=10$. We can see that for a range of values for $\mu, T_{n}$ and $S_{n}$ exhibit chaotic behavior, while $I_{n}$ does not. This also occurs for a range of values for $\beta$ in Figure ??, but it is interesting that $I_{n}$ bifurcates for different values of $\beta$ than $T_{n}$ and $S_{n}$, yet their bifurcations eventually seem to coincide. Here, $\alpha=5, \gamma=1, \mu=2.5, \sigma=3, S_{0}=5, I_{0}=10$. We should note that there were several hundred iterations performed for each figure, and it appears that this is sufficient to determine the convergence of the fixed points.
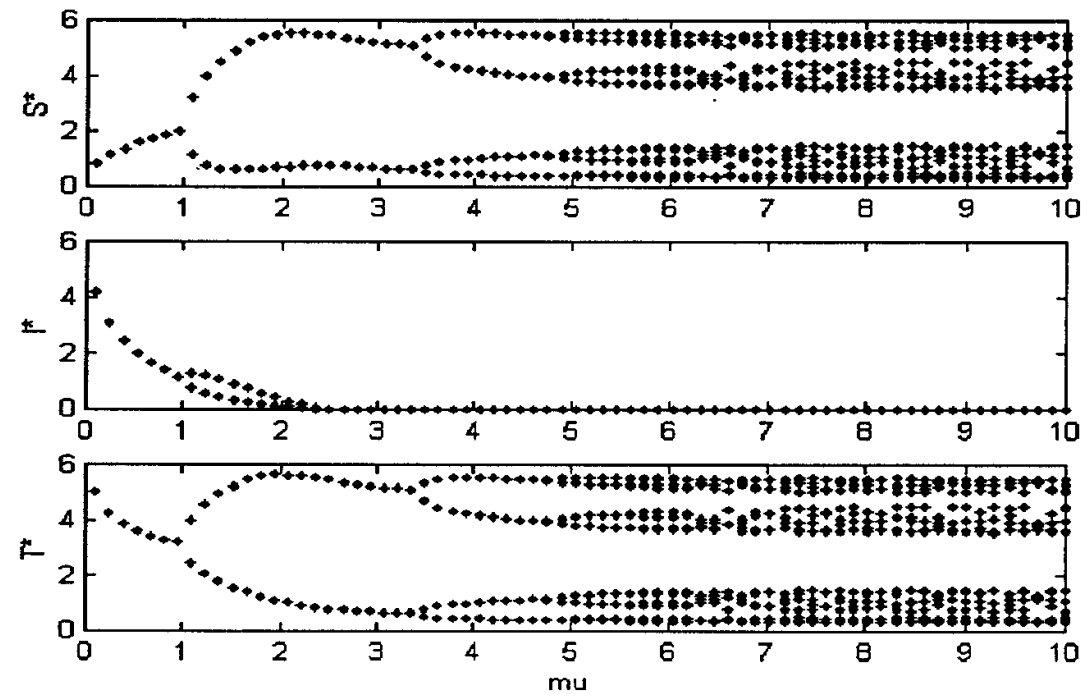

Figure 4: Bifurcation on $\mu: \alpha=5, \beta=15, \gamma=1, \sigma=0.1, S_{0}=5, I_{0}=10$.

These results are significant in that they show that the disease can exhibit dynamics independent from the demography. So, for many parameter values, the study of the 


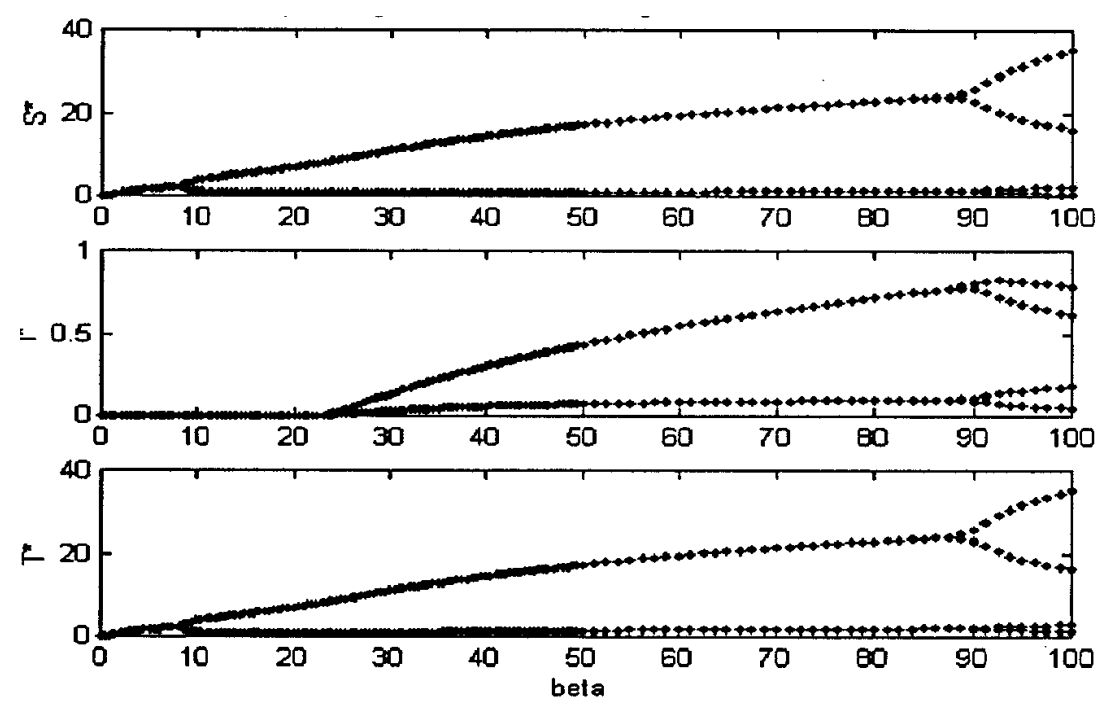

Figure 5: Bifurcation on $\beta: \alpha=5, \gamma=1, \mu=2.5, \sigma=3, S_{0}=5, I_{0}=10$.

demographic dynamics would not indicate the dynamics of the disease in the population. However, with limited resources, it is not possible for us to fully explore any bounds on the parameters in which we can determine the behavior of the system. These simulations serve merely as interesting examples of the dynamics we have found thus far.

\subsection{A strange attractor for the system?}

In iterating the system for a few initial conditions and fixed parameters we see what may be a strange attractor. In the plots to follow, $S_{n}$ is on the vertical axis and $I_{n}$ is on the horizontal axis. In the computations the parameters were fixed as follows: $\alpha=1.5, \beta=170, \gamma=2, \sigma=0.1, S_{o}=10, I_{o}=10$.

Figure 6 shows the last 35,000 iterates of 70,000 iterates. We believe that the iterates shown represent the shape of the attractor. This is because we know that for a these fixed parameters, $T_{\infty}$ is bounded above by about 29.4149. So, $S_{\infty}$ and $I_{\infty}$ must also be bounded above. The graphs show that no iterates go beyond $I_{n} \approx 30$. Figures $7 \& 8$ show iterates 34,000-35,000 and 69,000-70,000, respectively. We can see from these figures that after roughly 35,000 iterates, the maximum $S_{n}^{\prime} s$ have increased. Because of the bounds on $T_{n}$ and $I_{n}, S_{n}$ must be bounded above by around 0.5 . We can see that all iterates must be trapped within the crude shape that forms in figure 6 . After a huge number of iterates (the transients only comprise about five of the 70,000 iterates), the attractor will show its true form. More numerical simulations will give us more insight as to whether it is indeed a strange attractor. 


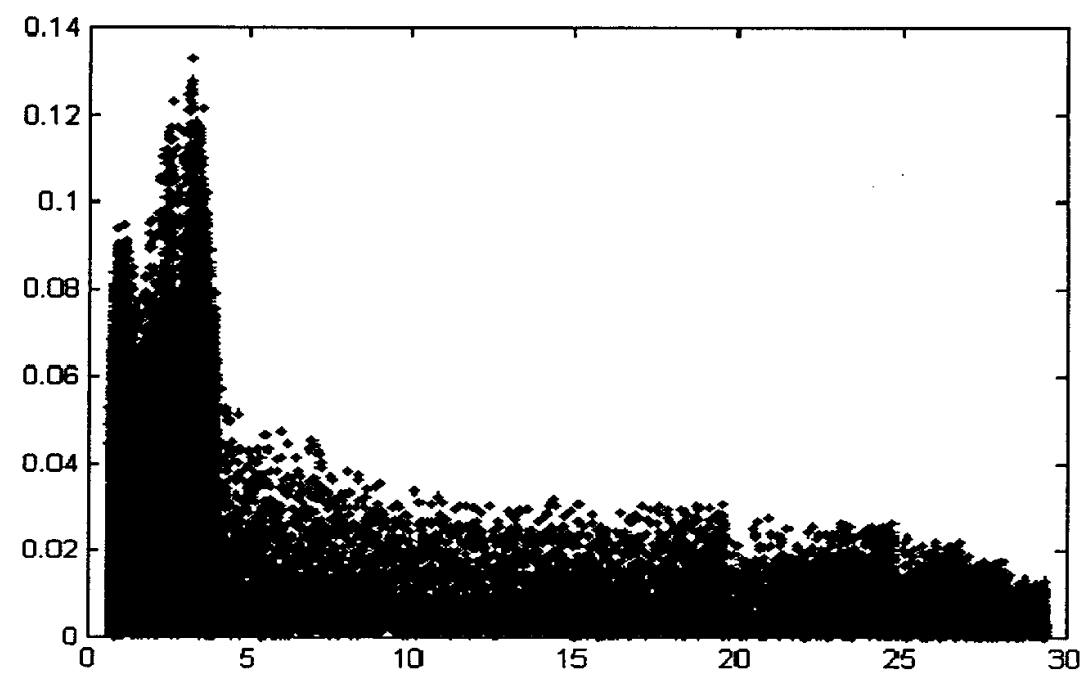

Figure 6: A strange attractor?

\section{$4 \rho>0$ : death due to infection}

This model takes into consideration that the infection can generate vital statistics. We take $\rho>0$

$$
\begin{aligned}
S_{n+1} & =\beta T_{n} e^{-\gamma T_{n}}+S_{n} e^{-\alpha I_{n}} e^{-\mu}+e^{-\mu}\left[1-e^{-\sigma}\right] I_{n} \\
I_{n+1} & =S_{n} e^{-\mu}\left[1-e^{-\alpha I_{n}}\right]+I_{n} e^{-\mu} e^{-\sigma} e^{-\rho} \\
T_{n+1} & =S_{n+1}+I_{n+1}=\beta T_{n} e^{-\gamma T_{n}}+T_{n} e^{-\mu}+I_{n} e^{-(\mu+\sigma)}\left(e^{-\rho}-1\right)
\end{aligned}
$$

Computer simulations were used to analyze this system (without a $T_{\infty}$ substitution). Figure (9) shows a bifurcation plot with varying $\rho$. Here, $\alpha=1.5, \beta=170, \gamma=2, \mu=$ $2, \sigma=0.1, S_{0}=10, I_{0}=10$. We can see results similar to that of varying $\mu$. We would need to run further simlations, but we can conclude that changes in death rates (due to natural causes, or infection) drastically affect the dynamics of the system and the infected population again exhibits dynamics independent from the demography.

\section{Conclusion}

Our analysis has shown that we can make definitive conclusions about the stability of certain disease levels in our SIS model. We have proven that if the infection rate per infective $\left(R_{0}\right)$ can be estimated to be less than one, the infection does not persist. If the infection rate per infective is greater than one, the infection persists in the population.

When we consider a non-constant recruitment (or birth) rate, the stability of the demographics is entirely dependent on the average number of children an individual has during a lifetime, $R_{d}$. The previous results of stability for the infective population apply, as 


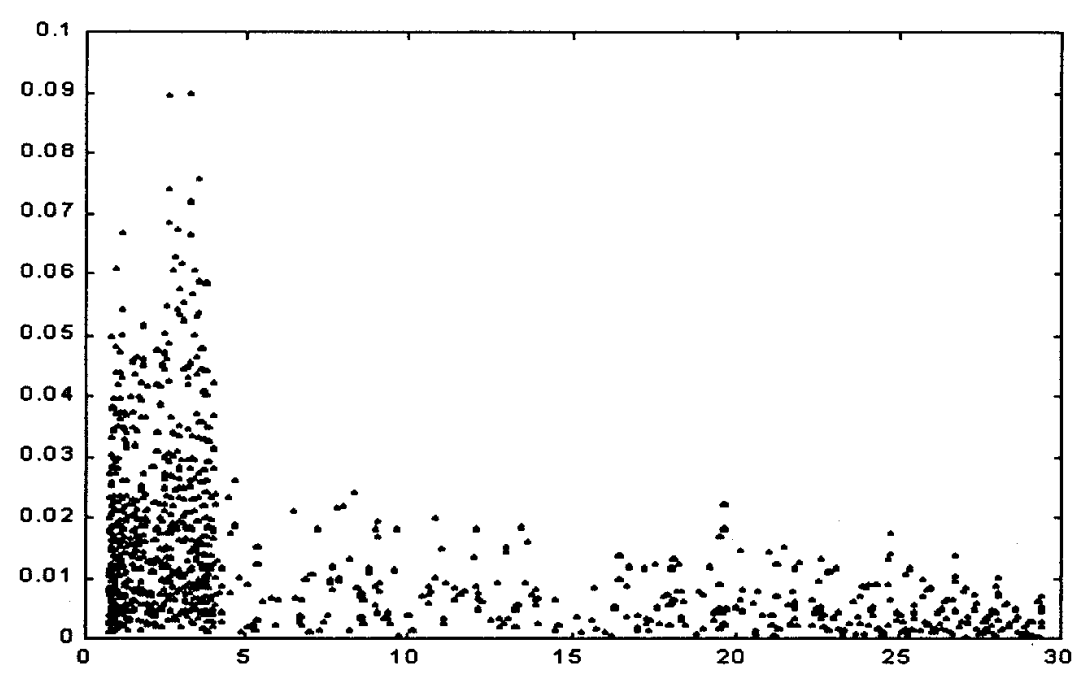

Figure 7: Iterates 34,900-35,000, maximum $S_{n} \approx 0.09$

for constant recruitment, when $R_{d}$ is in a range such that there is one limiting demographic value.

Through computer simulation, we discovered that the model with Ricker recruitment exhibits chaotic dynamics for values of $R_{d}$ in certain ranges. We have shown strong evidence for the existence of a strange attractor. Our simulations may show mathematically interesting results for period-doubling limiting demographic values; however, this would of course require further analysis before any significant conclusions could be made about the biological implications.

The main goal of this project was to develop a general SIS difference equation model that could be applied to different diseases and to analyze the stability of different infectious states in the model. We would hope that the model has been kept general enough so that it may be used with biologically significant parameter values for various diseases, and that our results could provide a preliminary analysis of the epidemic.

\section{References}

[1] Linda J. S. Allen and Amy M. Burgin, Comparison of Deterministic and Stochastic SIS and SIR Models, Technical Report No. 98-003, (1998)

[2] L. J. S. Allen, Some Discrete-Time SI, SIR, and SIS Epidemic Models, Math. Bios., 124 (1994), pp. 83-105.

[3] R. M. Anderson and M. May, Infectious Diseases of Humans, Oxford University Press, Oxford (1991).

[4] Carlos Castillo-Chavez and Horst R. Theime, Asymptotically Autonomous Epidemic Models. 


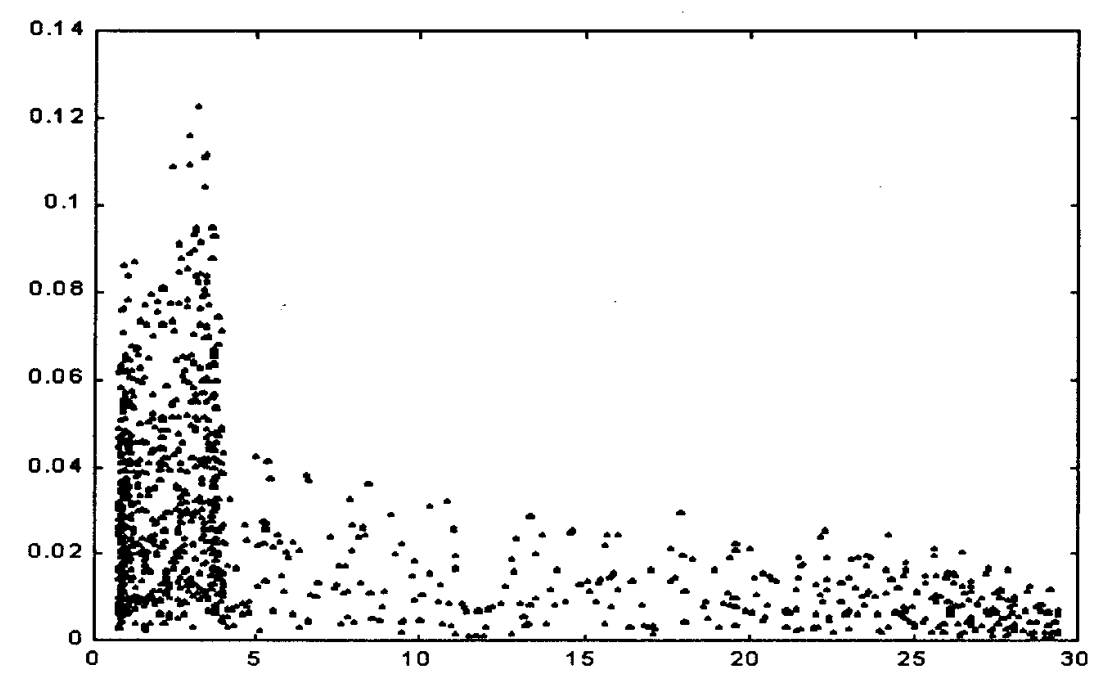

Figure 8: Iterates $69,000-70,000$, maximum $S_{n} \approx 0.125$

[5] P. Cull, Stability of Discrete One-Dimensional Population Models, Bul. Math. Biol., 50 (1988), pp. 67-75.

[6] P. Cull, Local and Global Stability for Population Models, Biol. Cybern., 54 (1986), pp. 141-149.

[7] P. Cull, Global Stability of Population Models, Bul. Math. Biol., 43 (1981), pp. 47-58.

[8] L. Edelstein-Keshet, Mathematical Models in Biology, The McGraw-Hill Co., Massachussets, (1988).

[9] J.Guckenheimer and Phillip Holmes, Nonlinear Oscillations, Dynamical Systems, and Bifurcations of Vector Fields. Springer-Verlag, New York, (1997)

[10] J. Jacquez, C. Simon and J. Koopman, Core Groups and $R_{0} s$ for Subgroups in Heterogeneous SIS and SI Models, Epidemic Models: Their Structure and Relation to Data, Cambridge University Press, New York, (1995), pp. 279-301.

[11] C. C. McCluskey and J.S. Muldowney, Bendixson-Dulac Criteria for Difference Equations, J. Dyn. Diff. Eq., 10 (1988), pp. 567-576.

[12] S.H. Strogatz, Nonlinear Dynamics and Chaos. Addison-Wesley Publishing Co., Massachussets, (1995)

\section{Acknowledgements}

This study was supported by the following institutions and grants: National Science Foundation (NSF Grant DMS 9977919); National Security Agency (NSA Grant MDA 

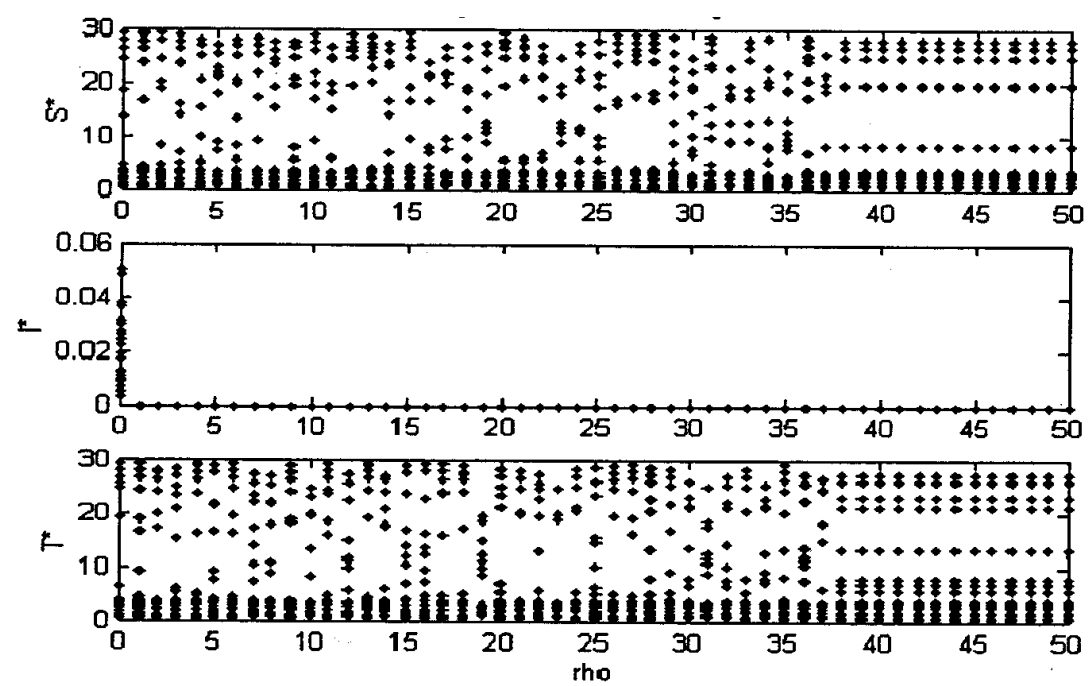

Figure 9: Bifurcation on $\rho: \alpha=1.5, \beta=170, \gamma=2, \mu=2, \sigma=0.1, S_{0}=10, I_{0}=10$.

9149710074); Presidential Faculty Fellowship Award (NSF Grant DEB 925370) and Presidetial Mentoring Award (NSF Grant HRD 9724850) to Carlos Castillo-Chavez; the office of the provost of Cornell University; Intel Technology for Education 200 Equipment Grant. Special thanks to Ted Greenwood of the Sloan Foundation. We also want to thank our advisors Abdul-Aziz Yakubu, and Carlos Castillo-Chavez for their academic and moral support. Special thanks to everybody that participated in MTBI 1999. 